\title{
Expressibility and trainability of parametrized analog quantum systems for machine learning applications
}

\author{
Jirawat Tangpanitanon, ${ }^{1, *}$ Supanut Thanasilp, ${ }^{1}$ Ninnat Dangniam, ${ }^{2,3}$ Marc-Antoine Lemonde, ${ }^{1}$ and Dimitris G. Angelakis ${ }^{1,4, \dagger}$ \\ ${ }^{1}$ Centre for Quantum Technologies, National University of Singapore, 3 Science Drive 2, Singapore 117543 \\ ${ }^{2}$ Department of Physics and Center for Field Theory and Particle Physics, Fudan University, Shanghai 200433, China \\ ${ }^{3}$ State Key Laboratory of Surface Physics, Fudan University, Shanghai 200433, China \\ ${ }^{4}$ School of Electrical and Computer Engineering, Technical University of Crete, Chania 73100 Greece
}

(Received 5 June 2020; accepted 25 November 2020; published 14 December 2020)

\begin{abstract}
Parametrized quantum evolution is the main ingredient in variational quantum algorithms for near-term quantum devices. In digital quantum computing, it has been shown that random parametrized quantum circuits are able to express complex distributions intractable by a classical computer, leading to the demonstration of quantum supremacy. However, their chaotic nature makes parameter optimization challenging in variational approaches. Evidence of similar classically intractable expressibility has been recently demonstrated in analog quantum computing with driven many-body systems. A thorough investigation of trainability of such analog systems is yet to be performed. In this work we investigate how the interplay between external driving and disorder in the system dictates the trainability and expressibility of interacting quantum systems. We show that if the system thermalizes, the training fails at the expense of the large expressibility, while the opposite happens when the system enters the many-body localized (MBL) phase. From this observation we devise a protocol using quenched MBL dynamics which allows accurate trainability while keeping the overall dynamics in the quantum supremacy regime. Our work shows the fundamental connection between quantum many-body physics and its application in machine learning. We conclude our work with an example application in generative modeling employing a well studied analog many-body model of a driven Ising spin chain. Our approach can be implemented with a variety of available quantum platforms including cold ions, atoms, and superconducting circuits.
\end{abstract}

DOI: 10.1103/PhysRevResearch.2.043364

\section{INTRODUCTION}

The recent achievement of quantum supremacy [1], the ability of quantum systems to compute tasks that are intractable by a classical computer, stands as an important milestone for noisy intermediate-scale quantum (NISQ) devices [2]. A common approach to operate NISQ devices is to implement variational quantum algorithms (VQAs), where a classical feedback loop is used to passively correct the noise in the quantum device [3-5]. VQAs have been implemented to tackle a wide range of problems, from quantum chemistry [6-11], machine learning [12,13], quadratic binary optimization [14-16], to high energy physics [17].

One of the key questions for NISQ devices is whether they can provide provable quantum advantage for real-world problems. A hint to answer this question lies in the ability of NISQ devices to efficiently explore Hilbert space. For example, in quantum chemistry, NISQ devices can produce highly entangled variational ansatzes, such as unitary coupled

\footnotetext{
*cqtjt@nus.edu.sg

†dimitris.angelakis@nus.edu.sg
}

Published by the American Physical Society under the terms of the Creative Commons Attribution 4.0 International license. Further distribution of this work must maintain attribution to the author(s) and the published article's title, journal citation, and DOI. clusters, that cannot be efficiently represented on a classical computer [18]. In machine learning, quantum circuits have been proven to have more "expressive power" than any classical neuron networks [19-21]. This means that those circuits can produce complex probability distributions that cannot be efficiently sampled from a classical computer.

Similar to classical variational algorithms, VQAs rely on "good" ansatzes that can efficiently capture the answer of a given problem. In the case when such ansatz is not known or implementable, it is desirable to exploit high expressibility of some NISQ devices to generate an unbiased guess. The latter is known as "hardware efficient" [8]. A common feature of this approach is to explore the chaotic dynamics which allows the system to quickly explore the entire Hilbert space. However, this chaoticity also makes it difficult, if not impossible, to classically optimize the system since it is highly sensitive to any small changes in the parameters. In the digital case, hardware efficient ansatzes suffer from the barren plateaus problem [22,23], where the landscape of the cost function becomes exponentially flat as the number of qubits increases. Hence, finding the right VQA for a given problem is an emerging art of balancing expressibility, implementability, and trainability of the NISQ devices.

Analog quantum simulators stand out from their digital counterpart when it comes to implementability [24-26]. Here, a quantum device is built to mimic a specific Hamiltonian, which requires significantly less control than universal quantum circuits. State-of-the-art quantum simulators have 
already been able to produce dynamics intractable by existing classical algorithms [27]. Quantum supremacy in analog simulators have also been proven in two-dimensional (2D) Ising lattice [28,29], cluster states [30], and more recently in periodically driven quantum many-body systems [31]. Hybrid analog-digital approaches for VQAs have been explored in Refs. [7-9,13,14,32-34].

In this work we analyze the expressibility and trainability of analog quantum devices focusing on parametrized driven quantum many-body systems. We show that these properties are intimately related to phases of the system. We focus on four generic phases depending on whether the dynamics is thermalized or many-body localized (MBL) $[35,36]$ and whether a continuous drive is applied. As an example, we consider the standard Ising chain, globally driven by an external magnetic field. We find that, evolving under the dynamics resulting from a series of quenches between randomized disorder configurations, the system in all four phases are capable of reaching the quantum supremacy regime, illustrating its high expressibility beyond a classical computer. We then devise a simple sequential training protocol to train the system for generative modeling tasks in machine learning. We show that the chaoticity in the thermalized phase prevents the training as in the digital case. However, the integrability of the MBL within each quench increases drastically the trainability of the system. The final learning accuracy depends solely on the phase of the system.

\section{DRIVEN ANALOG QUANTUM SYSTEMS AND THEIR STATISTICS}

In this section we study the many-body dynamics of generic parametrized quantum systems and the different statistics associated with their phases. We then analyze a specific example of driven quantum Ising chain which will be used for the analysis of the expressibility and trainability in the following sections.

\section{A. General framework}

We consider fully general quenched quantum many-body systems $\left|\psi\left(\underline{\Theta}_{M}\right)\right\rangle=\hat{\mathcal{U}}\left(\underline{\Theta}_{M}\right)\left|\psi_{0}\right\rangle$, where $\left|\psi_{0}\right\rangle$ is an initial product state, $\underline{\Theta}_{M}$ is a vector containing all variational parameters during the evolution, and $M$ is the number of times the system is quenched. The unitary time evolution is

$$
\hat{\mathcal{U}}\left(\underline{\Theta}_{M}\right)=\hat{U}\left(\underline{\theta}_{M}\right) \hat{U}\left(\underline{\theta}_{M-1}\right) \cdots \hat{U}\left(\underline{\theta}_{1}\right),
$$

where $\underline{\Theta}_{M}=\left\{\underline{\theta}_{m}\right\}_{m=1}^{M}$ and each quench/layer is obtained from a time-dependent Hamiltonian $\hat{H}\left(\underline{\theta}_{m}, t\right)$, i.e.,

$$
\hat{U}\left(\underline{\theta}_{m}\right)=\hat{\mathcal{T}} \exp \left(-i \int_{0}^{T} \hat{H}\left(\underline{\theta}_{m}, t\right) d t\right),
$$

with $m \in\{1,2, \ldots, M\}, \hat{\mathcal{T}}$ being the time-ordering operator, and $T$ being the evolution time during each layer. The Hamiltonian is further decomposed as

$$
\hat{H}\left(\underline{\theta}_{m}, t\right)=\hat{H}_{0}\left(\underline{\theta}_{m}\right)+f(t) \hat{V},
$$

where $\hat{H}_{0}\left(\underline{\theta}_{m}\right)$ is a static Hamiltonian, and $\hat{V}$ is the driving Hamiltonian such that $\left[\hat{H}_{0}\left(\underline{\theta}_{m}\right), \hat{V}\right] \neq 0$. The modulation $f(t)$ is an oscillating function with the period $T$. We require that the time-averaged Hamiltonian $\hat{H}_{\text {ave }}\left(\underline{\theta}_{m}\right)=\frac{1}{T} \int_{0}^{T} \hat{H}\left(\underline{\theta}_{m}, t\right) d t$ is many body [37].

\section{B. The four phases of $\hat{U}\left(\theta_{m}\right)$}

In the following we will refer to $\hat{U}\left(\underline{\theta}_{m}\right)$ as "thermalized" if any observations made on $\left|\psi_{M^{\prime}}^{\prime}\right\rangle=\hat{U}\left(\underline{\theta}_{m}\right)^{M^{\prime}}\left|\psi_{0}\right\rangle$ with $M^{\prime} \rightarrow \infty$ can be obtained from the microcanonical ensemble predictions associated with the energy $\bar{E}\left(\underline{\theta}_{m}\right) \pm \Delta E$, where $\bar{E}\left(\underline{\theta}_{m}\right)=\left\langle\psi_{0}\left|\hat{H}_{\mathrm{eff}}\left(\underline{\theta}_{m}\right)\right| \psi_{0}\right\rangle$. The effective Hamiltonian $\hat{H}_{\text {eff }}\left(\underline{\theta}_{m}\right)$ is defined such that $\hat{U}\left(\underline{\theta}_{m}\right) \equiv \exp \left[-i \hat{H}_{\text {eff }}\left(\underline{\theta}_{m}\right) T\right]$. Most quantum many-body systems follow this property according to the eigenstate thermalization hypothesis (ETH) [38]. Likewise, we refer to $\hat{U}\left(\underline{\theta}_{m}\right)$ as "many-body localized" if the above is not true due to large disorder [39]. Partial experimental signatures of the MBL have been observed in cold neutral atoms [27,40-43], superconducting circuits [44,45], and trapped ions [46].

We can now define the four regimes or "phases" of $\hat{U}\left(\underline{\theta}_{m}\right)$ in the above sense according to whether the dynamics is thermalized or MBL and whether $f(t)$ is zero or nonzero. To allow nontrivial dynamics within each layer, we require $2 \pi / T$ to be smaller than a typical energy gap of $\hat{H}_{\text {ave }}\left(\underline{\theta}_{m}\right)$. We assume that all $\hat{U}\left(\underline{\theta}_{m}\right)$ 's in $\hat{\mathcal{U}}\left(\underline{\Theta}_{M}\right)$ belong to the same phase for simplicity.

Let us explore the various statistics associated with the four phases, starting with the $f(t)=0$ case in which $\hat{H}_{\text {eff }}\left(\underline{\theta}_{m}\right)=\hat{H}_{\text {ave }}\left(\underline{\theta}_{m}\right)$. For the thermalized dynamics, the statistics of $\hat{H}_{\text {ave }}\left(\underline{\theta}_{m}\right)$ follows the Gaussian orthogonal ensemble (GOE) [39]. This is the ensemble of matrices whose entries are independent normal random variables subjected to the orthogonality constraint. This randomness is a signature of quantum chaos, which is a crucial ingredient for thermalization [39]. A large disorder can prevent the system from thermalization leading to MBL dynamics. In this case, the eigenenergies of $\hat{H}_{\text {ave }}\left(\underline{\theta}_{m}\right)$ follow the Poisson (POI) statistics, indicating that they are uncorrelated.

In the driven case, i.e., $f(t) \neq 0$, the statistics are defined at the level of the unitary operator $\hat{U}\left(\underline{\theta}_{m}\right)$, as it is generally not possible to have access to $\hat{H}_{\text {eff }}$. For the driven thermalized dynamics, the statistics of $\hat{U}\left(\underline{\theta}_{m}\right)$ follows the circular orthogonal ensemble (COE) [47]. This is the ensemble of matrices whose entries are independent complex normal random variables subjected to the orthogonality and the unitary constraints. Unlike the GOE, the COE is intimately related to the infinite-temperature ensemble and is not possible to obtain without a drive [47]. As before, a large disorder can prevent thermalization even with $f(t) \neq 0$, leading to the POI statistics of the quasienergies (to be defined later) $[48,49]$. A summary of all the statistics is given in Table I.

\section{Driven disordered quantum Ising chains}

To illustrate the four generic phases, we will work on a specific example of driven quantum Ising chains with

$$
\begin{gathered}
\hat{H}_{0}\left(\underline{\theta}_{m}\right)=\sum_{i=1}^{L} \theta_{i, m} \hat{Z}_{i}+J \sum_{i=1}^{L-1} \hat{Z}_{i} \hat{Z}_{i+1}+\frac{h}{2} \sum_{i=1}^{L} \hat{X}_{i}, \\
\hat{V}=\sum_{i=1}^{L} \hat{X}_{i},
\end{gathered}
$$


TABLE I. A summary of statistics, expressibility, and trainability in the four regimes, defined by whether $\hat{U}\left(\underline{\theta}_{m}\right)$ is thermalized or MBL and whether $f(t)=0$ or $f(t) \neq 0$. The symbol "-" indicates that the statistics is not defined.

\begin{tabular}{lcccc}
\hline \hline & \multicolumn{2}{c}{$f(t)=0$} & & $f(t) \neq 0$ \\
\cline { 2 - 4 } Features & Thermalized & MBL & Thermalized \\
\hline Statistics of $\hat{H}$ ave $\left(\underline{\theta}_{m}\right)$ & GOE & POI & - & MBL \\
Statistics of $\hat{U}\left(\underline{\theta}_{m}\right)$ & - & - & COE \\
Statistics of $\hat{\mathcal{U}}\left(\underline{\Theta}_{M}\right)$ with $M \gg 1$ & CUE & CUE & CUE \\
High expressibility (quantum supremacy) & yes & yes & yes \\
Trainability for generative modeling & no & yes & no \\
\hline \hline
\end{tabular}

where $f(t)=-\frac{F}{2} \cos (\omega t), \omega=2 \pi / T, \underline{\theta}_{m}=\left\{\theta_{i, m}\right\}_{i=1}^{L}, L$ is the number of spins, $\left\{\hat{X}_{i}, \hat{Z}_{i}\right\}$ are Pauli's operators acting on site $i, J$ is the interaction strength, $h$ is a static magnetic field, and $F$ is the driving amplitude. The parameters $\left\{\theta_{i, m}\right\}$ are "varied" by randomly drawing them from a uniform distribution in the range $[0, W]$ where $W$ is the disorder strength. This allows us to vary the parameters without changing the phase of the system. The dimension of the Hilbert space is $N=2^{L}$. The initial state $\left|\psi_{0}\right\rangle$ is prepared as a product state where each spin points along the $+z$ direction. This simple model has been implemented in various quantum platforms, including Rydberg atoms [50], trapped ions [51], and superconducting circuits [16].

The standard way to analyze the statistics of the system is to define the level statistics $\operatorname{Pr}\left(r_{\alpha}\right)$ as the normalized distribution of

$$
r_{\alpha} \equiv \frac{\min \left(\Delta_{\alpha+1}, \Delta_{\alpha}\right)}{\max \left(\Delta_{\alpha+1}, \Delta_{\alpha}\right)},
$$

where $\Delta_{\alpha}=\mathcal{E}_{\alpha+1}-\mathcal{E}_{\alpha}$ is the level spacing with $\mathcal{E}_{\alpha+1}>\mathcal{E}_{\alpha}$ and $\alpha=1,2, \ldots, 2^{L}-1$. In the $f(t)=0$ case, $\left\{\mathcal{E}_{\alpha}\right\}$ are eigenenergies of $\hat{H}_{\text {ave }}\left(\underline{\theta}_{m}\right)$. In the $f(t) \neq 0$ case, $\left\{\mathcal{E}_{\alpha}\right\}$ are quasienergies, defined such that $\left\{\exp \left(-i \mathcal{E}_{\alpha} T\right)\right\}$ are eigenvalues of $\hat{U}\left(\underline{\theta}_{m}\right)$. Not only that $H_{\text {eff }} \neq H_{\text {ave }}$ in the driven case, but the quasienergies are also defined in the limited range $\mathcal{E}_{\alpha} \in[0,2 \pi)$. This energy folding has profound impact on the resulting statistic.

In Fig. 1 we show the level statistics for $F=0$ and $F=$ $2.5 J$ with $W=1 J$ and $W=20 J$. For a small disorder $W=$ $1 J$, the level statistics of $\hat{H}_{\text {ave }}\left(\underline{\theta}_{m}\right)$ and $\hat{U}\left(\underline{\theta}_{m}\right)$ agree with the predictions from the GOE and the COE, respectively. For a large disorder $W=20 \mathrm{~J}$, the level statistics of both $\hat{H}_{\text {ave }}\left(\underline{\theta}_{m}\right)$ and $\hat{U}\left(\underline{\theta}_{m}\right)$ follows the POI distribution, as expected.

\section{EXPRESSIBILITY OF DRIVEN QUANTUM MANY-BODY SYSTEMS}

In this section we show that, given a large number of quenches $M$, the overall dynamics described by $\hat{\mathcal{U}}\left(\underline{\Theta}_{M}\right)$ for all four phases is capable of reaching the quantum supremacy regime, implying high expressibility of our system beyond a classical computer.

\section{A. Expressibility and quantum supremacy}

Expressibility is the term used in machine learning to describe the range of the resulting functions that a model can compute [52]. Exact measures for expressibility usually differ depending on the task. In the context of quantum computing, expressibility usually relates to how much a quantum system can explore the Hilbert space [53]. For example, product state ansatz have a lower expressibility than tensor-network ansatz, due to their inability to capture entangled states [54]. Specifically, the measure of expressibility proposed in [53] is related to the frame potential which measures how far a given unitary ensemble is from $t$ design [55]. However, the number of unitaries in the ensemble required to show $t$ design grows exponentially with $L$, making it impractical for large systems.

In this work we say that a quantum model has a higher expressibility than any classical models if there exists a probability distribution $p\left(\underline{z} ; \underline{\Theta}_{M}\right)$ that cannot be efficiently sampled from a classical computer unless the polynomial hierarchy collapses. This situation is referred to as quantum supremacy. In random quantum circuit proposals for quantum supremacy, a universal set of quantum gates are designed such that the system is chaotic and follows $t$ design [56]. Hence, these quantum circuits produce the highest expressibility according to the measure proposed in Ref. [53].

To understand the requirements for quantum supremacy, let us consider the task of approximating $p\left(z ; \Theta_{M}\right)$ up to additive error, i.e.,

$$
\sum_{\underline{z} \in\{0,1\}^{L}}\left|p\left(\underline{z} ; \underline{\Theta}_{M}\right)-q(\underline{z})\right| \leqslant v
$$

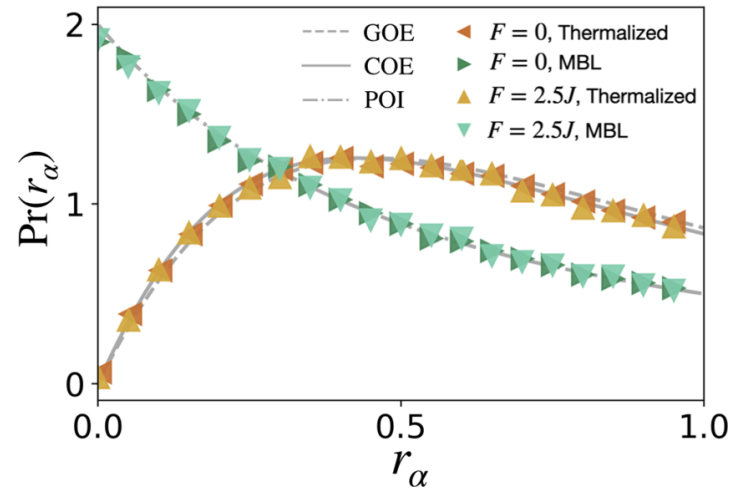

FIG. 1. Level statistics of $\hat{H}_{\text {ave }}\left(\underline{\theta}_{m}\right)$ for $f(t)=0$ and $\hat{U}\left(\underline{\theta}_{m}\right)$ for $F=2.5 J$. The thermalized and the MBL phases are obtained with $W=1 J$ and $W=20 J$, respectively. $(L=9, \omega=8 J, h=2.5 J, 500$ disorder realizations.) 

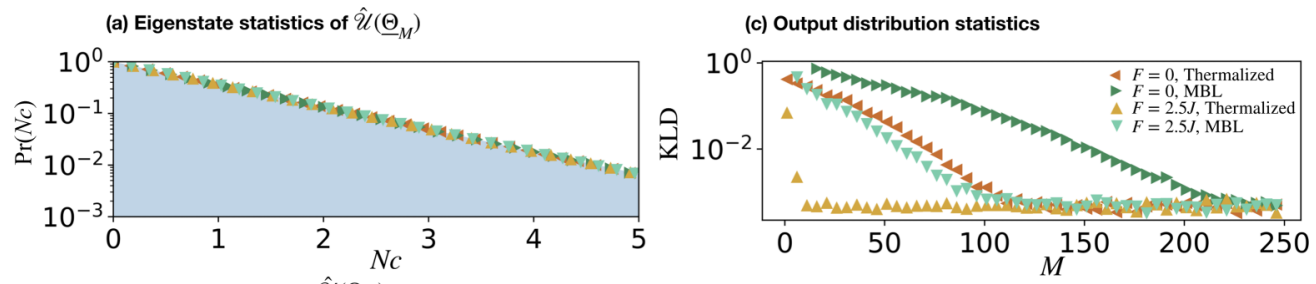

(b) Level statistics of $\hat{\mathscr{U}}\left(\underline{\Theta}_{M}\right)$


FIG. 2. Statistics of parametrized analog quantum many-body evolution: (a) and (b) The eigenstate distribution $\operatorname{Pr}(N c)$ and the level statistics $\operatorname{Pr}\left(r_{\alpha}\right)$ for the four phases of $\hat{U}\left(\underline{\theta}_{m}\right)$, respectively, with $M=400$. The shaded areas are the predictions from the CUE statistics. (c) The KLD of the output distribution from the Porter-Thomas distribution as a function of $M$. (d) The KLD of $p\left(z ; \underline{\Theta}_{m+\delta m}\right)$ from $p\left(\underline{z} ; \underline{\Theta}_{m}\right)$ as a function of $\delta m$. The KLD is averaged over $M \in[378,400)$ for a given $\delta m$. The thermalized and and the MBL phases are obtained with $W=1 J$ and $W=20 J$, respectively. ( $L=9, \omega=8 J, h=2.5 J, 500$ disorder realizations).

where $v$ is a positive constant, $\{z\}$ are output bit strings measured in the computational basis, $p\left(z ; \Theta_{M}\right)=\left|\left\langle z \mid \psi\left(\Theta_{M}\right)\right\rangle\right|^{2}$ is the exact output probability, and $q(\underline{z})$ is the approximated value obtained from a classical / quantum device. In principle, a quantum device can satisfy this condition by directly implementing $\hat{\mathcal{U}}\left(\underline{\Theta}_{M}\right)$ in the hardware and measure the output multiple times to construct $q(\underline{z})$. To show that a classical computer cannot do the same efficiently unless the polynomial hierarchy collapses, one need to show that (i) it is \#P-hard to approximate $p\left(z ; \underline{\Theta}_{M}\right)$ up to multiplicative error [57], i.e.,

$$
\left|p\left(\underline{z} ; \underline{\Theta}_{M}\right)-q(\underline{z})\right| \leqslant \eta p\left(\underline{z} ; \underline{\Theta}_{M}\right)
$$

for some $\eta$ and (ii) the output probability anticoncentrates [58], i.e.,

$$
\operatorname{Pr}\left(p\left(\underline{z} ; \underline{\Theta}_{M}\right)>\frac{\delta}{N}\right) \geqslant \gamma,
$$

where $\delta, \gamma$ are some constants. We refer interested readers to Refs. $[28,59,60]$ for the derivation of how these two conditions lead to the proof of quantum supremacy.

\section{B. Achieving quantum supremacy with quenched quantum many-body systems}

The \#P-hardness to approximate $p\left(z ; \underline{\Theta}_{M}\right)$ up to multiplicative error has been shown (for the worse instance) in the case where it results from a unitary evolution that follows the circular unitary ensemble (CUE) statistics [60,61]. The CUE is the ensemble of matrices whose entries are independent complex normal random variables subject to the unitary constraint [62]. Such statistics can be probed from both the previously defined level statistics $\operatorname{Pr}\left(r_{\alpha}\right)$ and the distribution $\operatorname{Pr}\left(c=\left|\left\langle\underline{z} \mid \mathcal{E}_{\alpha}\right\rangle\right|^{2}\right)$ of the eigenstates $\left|\mathcal{E}_{\alpha}\right\rangle$ of $\hat{\mathcal{U}}\left(\underline{\Theta}_{M}\right)$.

Figures 2(a) and 2(b) show the statistics of the eigenstates and the quasienergies of $\hat{\mathcal{U}}\left(\underline{\Theta}_{M}\right)$ in the four regimes at $M=400$, respectively. It can be seen that in all cases the results match with the CUE statistics, indicating the \#P-hardness to approximate the resulting $p\left(\underline{z} ; \underline{\Theta}_{M}\right)$ up to mul- tiplicative error. Our finding agrees with Ref. [63], which shows that random quenches in atomic Hubbard and spin models with long-range interactions lead to the $t$-design property. The $t$-design ensemble produces the CUE when $t \rightarrow \infty$ which happens in the long-time limit [64].

In Fig. 2(c) we plot the Kullback-Leibler (KL) divergence of the output distribution $\operatorname{Pr}(p)$ from the Porter-Thomas distribution $\operatorname{Pr}_{\mathrm{PT}}(p)=N e^{-N p}$. The latter implies that the system explores the entire Hilbert space. (Here we drop the argument $\underline{\Theta}_{M}$ for brevity.) The Porter-Thomas distribution satisfies the anticoncentration condition since $\operatorname{Pr}_{\mathrm{PT}}\left(p>\frac{1}{N}\right)=$ $\int_{N p=1}^{\infty} d(N p) e^{-N p}=1 / e$ [61]. From Fig. 2(c) it can be seen that the system in all four phases reaches the Porter-Thomas distribution over time with different timescales. The thermalized case with $F=2.5 \mathrm{~J}$ reaches it first at $M \sim 10$. The thermalized case with $F=0$ and the MBL case with $F=$ $2.5 J$ have a similar convergence rate and saturate at $M \sim 100$. The MBL with $F=0$ has the slowest rate and saturates at $M \sim 250$. This is expected as MBL dynamics localizes the system, while the drive $F$ "heats up" the system leading to delocalization.

Figures 2(a)-2(c) provide evidences that $\left|\psi\left(\Theta_{M}\right)\right\rangle$ cannot be efficiently approximated by a classical computer. This suggests that, for a large number of qubits, our system in all phases have higher expressibility than any classical models.

\section{TRAINABILITY OF DRIVEN ANALOG QUANTUM MANY-BODY SYSTEMS}

In the context of machine learning, having a model with large expressibility is necessary but not sufficient as the model also needs to be trainable. We here address the interplay between expressibility and trainability for the four generic phases of driven analog many-body systems discussed so far. Interestingly, we show that the external drive and the temporal correlations between different quenches in the MBL phase are the key ingredients to combine those two crucial characteristics. 
(a) Generative modeling

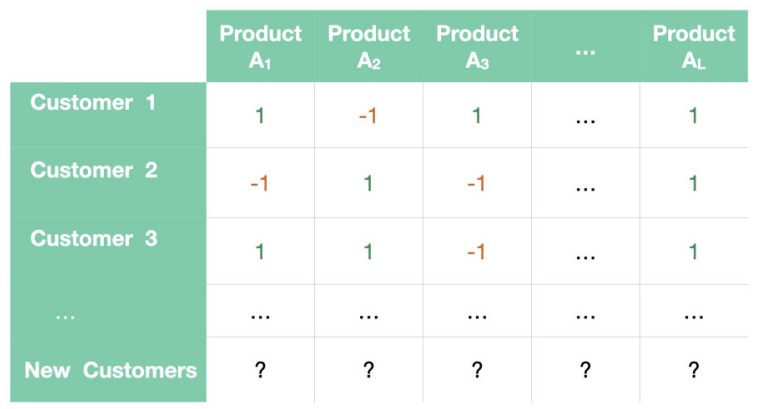

(b) Sequential training protocol

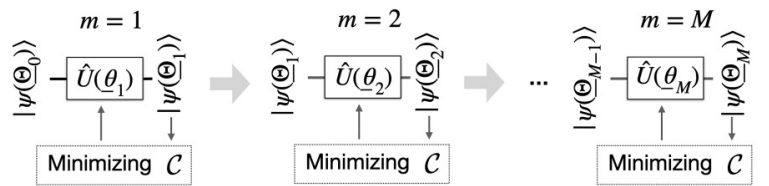

(c) Learning in the Hilbert space

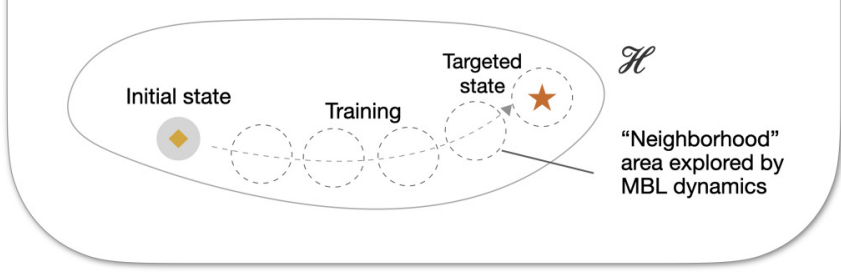

FIG. 3. Machine learning with a driven analog quantum processor: (a) A table demonstrating a real-world application of generative modeling tasks in machine learning. Each customer is asked to rate whether he/she likes $(+1)$ or dislikes $(-1)$ a given product. (b) A sketch of optimization loops used in the training protocol. (c) A diagram showing the movement of the system in the Hilbert space during the training in the MBL phase.

\section{A. Generative modeling in classical machine learning}

As a testbed to analyze the trainability of our model, we solve a generative modeling problem in machine learning [65]. The latter is an unsupervised task, meaning that the training data are unlabeled. The goal is to find the unknown probability distribution $Q(z)$ underlying the training data. Here the data is a set of binary vectors $\left\{\underline{z}_{\text {data }}=\left\{\underline{z}_{1}, \underline{z}_{2}, \ldots\right\}\right.$. For example, it can represent the opinions of a group of customers on a set of $L$ different products, as depicted in Fig. 3(a). The opinion of the customer $i$ is represented by a binary vector $\underline{z}_{i}=\left[z_{i 1}, z_{i 2}, \ldots, z_{i L}\right]$ where $z_{i j}=1$ if he/she likes the product $j$ and -1 otherwise. After knowing $Q(\underline{z})$, the company can generate new data from this distribution and recommend products with +1 score to new customers.

In this section we use an artificial data set as a working example. To assure the generality of the data, we assume that $Q(z)$ is the Boltzmann distribution of classical Ising spins with all-to-all connectivity, i.e.,

$$
Q(\underline{z})=\frac{1}{Z} e^{-E(\underline{z}) / k_{B} T_{0}},
$$

where $Z=\sum_{z} \exp \left[-E(\underline{z}) / k_{B} T_{0}\right]$ is the partition function, $k_{B}$ is the Boltzmann constant, $T_{0}$ plays the role of a temperature, and

$$
E(\underline{z})=\sum_{i=1}^{L} a_{i} z_{i}+\sum_{\langle i, j\rangle} b_{i j} z_{i} z_{j}
$$

with $a_{i}, b_{i j}$ being random numbers between $\pm J / 2$. This model is known as the Boltzmann machine which is one of the standard types of artificial neuron networks used in machine learning and has been shown to capture a wide range of real-world data [66]. Its quantum version has been studied in $[67,68]$.

\section{B. Sequential training scheme using an analog quantum model}

Classically, the distribution of $\{z\}_{\text {data }}$ can be obtained by first guessing a model $P_{\text {model }}(z ; \underline{\Theta})$, such as the Poisson or the Boltzmann distribution, which has some variational parameters $\Theta$. The "training" is done by minimizing the cost function, which is the KL divergence of $P_{\text {model }}(z ; \underline{\Theta})$ from $\tilde{Q}(z)$ using either gradient descent or gradient-free optimization algorithms. Here $\tilde{Q}$ is the normalized histogram of $\{z\}_{\text {data }}$.

In our case we show how the distribution of $\{\underline{z}\}_{\text {data }}$ can be recovered as the output probability $p\left(\underline{z} ; \underline{\Theta}_{M}\right)$ of the driven quantum Ising chain. This approach is also known as the Born's machine [21]. Our goal here is to guide or train the quantum system to a specific point in the Hilbert space such that $p\left(\underline{z} ; \underline{\Theta}_{M}\right)=Q(\underline{z})$. Our training protocol, depicted in Fig. 3(b), takes place as follows:

(1) Initialize the system at $\left|\psi\left(\underline{\Theta}_{m}\right)\right\rangle=\left|\psi_{0}\right\rangle$ with $m=0$ and $\underline{\Theta}_{0}=\{\}$.

(2) Evolve the system by one layer $\left|\psi\left(\underline{\Theta}_{m+1}\right)\right\rangle=$ $\hat{U}\left(\underline{\theta}_{m+1}\right)\left|\psi\left(\underline{\Theta}_{m}\right)\right\rangle$ with $\underline{\Theta}_{m+1}=\left\{\underline{\theta}_{m+1}\right\} \cup \underline{\Theta}_{m}$, and then measure $p\left(\underline{z} ; \underline{\Theta}_{m+1}\right)$ to compute $\mathcal{C}$.

(3) Repeat step $2 D$ times with different disorder realization $\underline{\theta}_{m+1}$, randomly chosen from $[0, W]$. In the thermalized case, the system will randomly explore the entire Hilbert space in this step. However, in the MBL case, the system will only explore the Hilbert space locally near $\left|\psi\left(\Theta_{m}\right)\right\rangle$ allowing systematic optimization, see Fig. 3(c).

(4) Choose the disorder realization in step 3 that minimizes $\mathcal{C}$, then update $m \rightarrow m+1$. This will "move" the state in the most promising direction in the Hilbert space.

(5) Repeat steps 3-4 until convergence.

We note here three characteristics of our training protocol. First, it is sequential since not all parameters in $\Theta$ are updated at the same time, making them easier for classical optimization. Second, although the parameters are randomly drawn during the training, our optimization is done systematically in the Hilbert space. This makes an important difference to the usual optimization approaches which are done in the parameter space [22,67]. Third, a large fraction of results is "thrown away" in step 3. Although in principle this data can be utilized to improve the training efficiency, it is our goal to keep the training protocol as simple as possible, so that the focus is made on distinct learning behaviors displayed by each phase. 




FIG. 4. Training analog quantum systems in the Hilbert space: (a) The lowest cost function at each training step $M$ for $F=0$ and $F=2.5 J$. The thermalized and and the MBL phases are obtained with $W=1 J$ and $W=20 J$, respectively. The shaded areas represent standard deviations. (b) The cost function at $M=10^{4}$ as a function of $W$. The results are averaged over 10 data set, i.e., 10 realizations of $\left\{a_{i}, b_{i}\right\}$ in Eq. (11). Each data set consists of 3000 samples. (c) The averaged level spacing $\left\langle r_{\alpha}\right\rangle$ at $M=10^{4}$ as a function of $W$. ( $L=9$, $\omega=8 J, h=2.5 J, k_{B} T_{0}=J$ and $\left.D=200\right)$.

\section{Training results}

The training results are shown in Fig. 4(a). As expected, the system in the thermalized phase cannot be trained. The cost function from the thermalized case with $F=2.5 \mathrm{~J}$ saturates at $C \sim 2$ already at the first layer. In the $F=0$ case, the cost function starts at around $C \sim 3.5$ and then falls down to saturate at $C \sim 2$, the same value as the driven case, when $M \sim 50$. For the MBL case with $F=0$, during $M \lesssim 10^{2}$, the cost function steadily decays to 0.7 . Then during $10^{2} \lesssim M \lesssim$ $10^{3}$, the cost function continues to decay with a slower rate. Interestingly, after $M \gtrsim 10^{3}$, the cost function increases and saturates at 0.7 when $M \sim 10^{4}$. In contrast, for the MBL case with $F=2.5 \mathrm{~J}$, the cost function goes down steadily when $M \lesssim 10$. Then the cost function further decays monotonically with a slower rate to saturate at $C \sim 0.1$ at $M \sim 10^{4}$. This results show that the learning behavior changes qualitatively depending on the phase and the timescale of the system. The best learning accuracy is obtained with the MBL phase with $F=2.5 \mathrm{~J}$.

In Fig. 4(b) we plot the final learning results as a function of $W$ for $F=0$ and $F=2.5 \mathrm{~J}$. For comparison, in Fig. 4(c), we also plot the averaged level spacing $\left\langle r_{\alpha}\right\rangle$ as a function of $W$ for both cases. In the $F=2.5 \mathrm{~J}$ case, the final learning accuracy shows a transition between the trainable and the untrainable regimes, which corresponds roughly to the phase transition between the CUE and the POI statistics. In the $F=0$ case, the system moves towards the trainability regime as $W$ approaches $30 J$. However, we stop our calculation here as the training takes too long to converge when $W>30 J$ [69]. Nevertheless, our present results are sufficient to conclude that the drive leads to a better learning accuracy for this timescale. We conjecture that, once the system in the undriven case fully reaches the trainable regime, the learning accuracy should monotonically decreases with $M$ until saturation.

Lastly, in machine learning, the ultimate goal is not just trainability but the capability of the model to make prediction, i.e., generalization. In addition, NISQ can only operate in a finite coherence time. To show both generalization and imple-

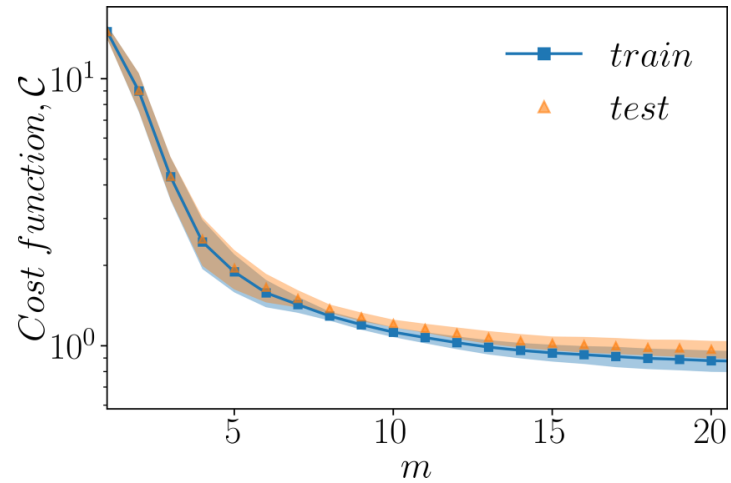

FIG. 5. The lowest cost function at each training step $M$ for $F=$ $2.5 \mathrm{~J}$ and $W=30 \mathrm{~J}$. The training data (2000 samples) and the testing data (1000 samples) are independently generated from Eq. (10). $\left(L=9, \omega=8 J, h=2.5 J, k_{B} T_{0}=J\right)$.

mentability of our model in the MBL phase with $F=2.5 \mathrm{~J}$, we train the model with 2000 training data sampled from the distribution in Eq. (10) using only up to $M=20$ cycles. In Fig. 5 we plot the KLD between $p\left(z ; \underline{\Theta}_{M}\right)$ and the testing distribution generated by 1000 testing data which are newly sampled from Eq. (10). We can see that the cost function converges for both the training and testing data set, indicating that the model does not overfit the data. We have also repeated the calculations in this section with a different data set drawn from the Bernouilli distributions [67] and similar results are obtained (not shown).

\section{Temporal correlations enabled by MBL}

To understand different learning accuracy in different phases, we calculate the KL divergence between $p\left(\underline{z} ; \underline{\Theta}_{M}\right)$ and $p\left(z ; \underline{\Theta}_{M+\delta m}\right)$ to measure the temporal correlations or the "memory" between outputs at different layers. In Fig. 2(d) we plot such KL divergence as a function of $\delta m$, averaged over various $M$ 's. In the thermalized phase, we find that there are no temporal correlations between layers. This is expected as each layer has chaotic dynamics which is highly sensitive to any small changes introduced to the system. In contrast, in the MBL phase, the system displays short-term memory that decays with $\delta m$. The MBL dynamics with $f(t)=0$ has the longest memory. This memory were exploited during the training to improve trainability of the system. We note that since at large $M$ the system produces a random state, the results in Fig. 2(d) can be equivalently obtained using short-time dynamics with $M=0$, but averaged over many random initial states.

\section{CONCLUSIONS}

In this work we have thoroughly analyzed the expressibility and trainability of parametrized analog quantum many-body systems. We show that both thermalized and MBL dynamics with and without the modulation $f(t)$ are capable of reaching the quantum supremacy regime, indicating high expressibility beyond any classical models. In the context of generative modeling, we show that chaoticity prevents systematic optimization of the system. However, the latter 
can be qualitatively improved by the MBL dynamics. In the future it would be interesting to analyze scalability and generalizability of our models as well as a more complex training protocol for efficient optimization.

\section{ACKNOWLEDGMENTS}

The authors gratefully acknowledge financial support through the National Research Foundation and Ministry of Education Singapore under the Research Centres of Excellence programme. It was also partially funded by Polisimulator project co-financed by Greece and the EU Regional Development Fund, the European Research Council under the European Union's Seventh Framework Programme (FP7/2007-2013). Ninnat Dangniam is supported by the National Natural Science Foundation of China (Grant No. 11875110).
[1] F. Arute, K. Arya, R. Babbush, D. Bacon, J. C. Bardin, R. Barends, R. Biswas, S. Boixo, F. G. S. L. Brandao, D. A. Buell, B. Burkett, Yu. Chen, Z. Chen, B. Chiaro, R. Collins, W. Courtney, A. Dunsworth, E. Farhi, B. Foxen, A. Fowler, C. Gidney, M. Giustina, R. Graff, K. Guerin, S. Habegger, M. P. Harrigan, M. J. Hartmann, A. Ho, M. Hoffmann, T. Huang, Travis S. Humble, S. V. Isakov, E. Jeffrey, Z. Jiang, D. Kafri, K. Kechedzhi, J. Kelly, P. V. Klimov, S. Knysh, A. Korotkov, F. Kostritsa, D. Landhuis, M. Lindmark, E. Lucero, D. Lyakh, S. Mandrà, J. R. McClean, M. McEwen, A. Megrant, X. Mi, K. Michielsen, M. Mohseni, J. Mutus, O. Naaman, M. Neeley, C. Neill, M. Y. Niu, E. Ostby, A. Petukhov, J. C. Platt, C. Quintana, E. G. Rieffel, P. Roushan, N. C. Rubin, D. Sank, Kevin J. Satzinger, V. Smelyanskiy, K. J. Sung, M. D. Trevithick, A. Vainsencher, B. Villalonga, T. White, Z. J. Yao, P. Yeh, A. Zalcman, H. Neven, and J. M. Martinis, Quantum supremacy using a programmable superconducting processor, Nature (London) 574, 505 (2019).

[2] J. Preskill, Quantum Computing in the NISQ era and beyond, Quantum 2, 79 (2018).

[3] M. Benedetti, E. Lloyd, S. Sack, and M. Fiorentini, Parameterized quantum circuits as machine learning models, Quantum Sci. Technol. 4, 043001 (2019).

[4] N. Moll, P. Barkoutsos, L. S. Bishop, J. M. Chow, A. Cross, D. J. Egger, S. Filipp, A. Fuhrer, J. M. Gambetta, M. Ganzhorn, A. Kandala, A. Mezzacapo, P. Müller, W. Riess, G. Salis, J. Smolin, I. Tavernelli, and K. Temme, Quantum optimization using variational algorithms on near-term quantum devices, Quantum Sci. Technol. 3, 030503 (2018).

[5] J. R. McClean, J. Romero, R. Babbush, and A. Aspuru-Guzik, The theory of variational hybrid quantum-classical algorithms, New J. Phys. 18, 023023 (2016).

[6] F. Arute, K. Arya, R. Babbush, D. Bacon, J. C. Bardin, R. Barends, S. Boixo, M. Broughton, B. B. Buckley, D. A. Buell, B. Burkett, N. Bushnell, Yu. Chen, Z. Chen, B. Chiaro, R. Collins, W. Courtney, S. Demura, A. Dunsworth, E. Farhi, A. Fowler, B. Foxen, C. Gidney, M. Giustina, R. Graff, S. Habegger, M. P. Harrigan, A. Ho, S. Hong, T. Huang, W. J. Huggins, L. Ioffe, S. V. Isakov, E. Jeffrey, Z. Jiang, C. Jones, D. Kafri, K. Kechedzhi, J. Kelly, S. Kim, P. V. Klimov, A. Korotkov, F. Kostritsa, D. Landhuis, P. Laptev, M. Lindmark, E. Lucero, O. Martin, J. M. Martinis, J. R. McClean, M. McEwen, A. Megrant, X. Mi, M. Mohseni, W. Mruczkiewicz, J. Mutus, O. Naaman, M. Neeley, C. Neill, H. Neven, M. Y. Niu, T. E. O'Brien, E. Ostby, A. Petukhov, H. Putterman, C. Quintana, P. Roushan, N. C. Rubin, D. Sank, K. J. Satzinger, V. Smelyanskiy, D. Strain, K. J. Sung, M. Szalay, T. Y. Takeshita, A. Vainsencher, T. White, N. Wiebe, Z. J. Yao, P. Yeh, and
A. Zalcman, Hartree-Fock on a superconducting qubit quantum computer, Science 369, 1084 (2020).

[7] C. Hempel, C. Maier, J. Romero, J. McClean, T. Monz, H. Shen, P. Jurcevic, B. P. Lanyon, P. Love, R. Babbush, A. Aspuru-Guzik, R. Blatt, and C. F. Roos, Quantum Chemistry Calculations on a Trapped-Ion Quantum Simulator, Phys. Rev. X 8, 031022 (2018).

[8] A. Kandala, A. Mezzacapo, K. Temme, M. Takita, M. Brink, J. M. Chow, and J. M. Gambetta, Hardware-efficient variational quantum eigensolver for small molecules and quantum magnets, Nature (London) 549, 242 (2017).

[9] Y. Li and S. C. Benjamin, Efficient Variational Quantum Simulator Incorporating Active Error Minimization, Phys. Rev. X 7, 021050 (2017).

[10] P. J. J. O’Malley, R. Babbush, I. D. Kivlichan, J. Romero, J. R. McClean, R. Barends, J. Kelly, P. Roushan, A. Tranter, N. Ding, B. Campbell, Y. Chen, Z. Chen, B. Chiaro, A. Dunsworth, A. G. Fowler, E. Jeffrey, E. Lucero, A. Megrant, J. Y. Mutus, M. Neeley, C. Neill, C. Quintana, D. Sank, A. Vainsencher, J. Wenner, T. C. White, P. V. Coveney, P. J. Love, H. Neven, A. Aspuru-Guzik, and J. M. Martinis, Scalable Quantum Simulation of Molecular Energies, Phys. Rev. X 6, 031007 (2016).

[11] A. Peruzzo, J. McClean, P. Shadbolt, M.-H. Yung, X.-Q. Zhou, P. J. Love, A. Aspuru-Guzik, and J. L. O'Brien, A variational eigenvalue solver on a photonic quantum processor, Nat. Commun. 5, 4213 (2014).

[12] D. Zhu, N. M. Linke, M. Benedetti, K. A. Landsman, N. H. Nguyen, C. H. Alderete, A. Perdomo-Ortiz, N. Korda, A. Garfoot, C. Brecque, L. Egan, O. Perdomo, and C. Monroe, Training of quantum circuits on a hybrid quantum computer, Sci. Adv. 5, eaaw9918 (2019).

[13] V. Havlíček, A. D. Córcoles, K. Temme, A. W. Harrow, A. Kandala, J. M. Chow, and J. M. Gambetta, Supervised learning with quantum-enhanced feature spaces, Nature (London) 567, 209 (2019).

[14] G. Pagano, A. Bapat, P. Becker, K. S. Collins, A. De, P. W. Hess, H. B. Kaplan, A. Kyprianidis, W. L. Tan, C. Baldwin, L. T. Brady, A. Deshpande, F. Liu, S. Jordan, A. V. Gorshkov, and C. Monroe, Quantum approximate optimization of the longrange Ising model with a trapped-ion quantum simulator, Proc. Natl. Acad. Sci. 117, 25396 (2020).

[15] F. Arute, K. Arya, R. Babbush, D. Bacon, J. C. Bardin, R. Barends, S. Boixo, M. Broughton, B. B. Buckley, D. A. Buell, B. Burkett, N. Bushnell, Y. Chen, Z. Chen, B. Chiaro, R. Collins, W. Courtney, S. Demura, A. Dunsworth, E. Farhi, A. Fowler, B. Foxen, C. Gidney, M. Giustina, R. Graff, S. Habegger, M. P. Harrigan, A. Ho, S. Hong, T. Huang, L. B. Ioffe, S. V. Isakov, E. Jeffrey, Z. Jiang, C. Jones, D. Kafri, K. 
Kechedzhi, J. Kelly, S. Kim, P. V. Klimov, A. N. Korotkov, F. Kostritsa, D. Landhuis, P. Laptev, M. Lindmark, M. Leib, E. Lucero, O. Martin, J. M. Martinis, J. R. McClean, M. McEwen, A. Megrant, X. Mi, M. Mohseni, W. Mruczkiewicz, J. Mutus, O. Naaman, M. Neeley, C. Neill, F. Neukart, H. Neven, M. Y. Niu, T. E. O'Brien, B. O'Gorman, E. Ostby, A. Petukhov, H. Putterman, C. Quintana, P. Roushan, N. C. Rubin, D. Sank, Kevin J. Satzinger, A. Skolik, V. Smelyanskiy, D. Strain, M. Streif, K. J. Sung, M. Szalay, A. Vainsencher, T. White, Z. J. Yao, P. Yeh, A. Zalcman, and L. Zhou, Quantum approximate optimization of non-planar graph problems on a planar superconducting processor, arXiv:2004.04197.

[16] J. S. Otterbach, R. Manenti, N. Alidoust, A. Bestwick, M. Block, B. Bloom, S. Caldwell, N. Didier, E. Schuyler Fried, S. Hong, P. Karalekas, C. B. Osborn, A. Papageorge, E. C. Peterson, G. Prawiroatmodjo, N. Rubin, Colm A. Ryan, D. Scarabelli, M. Scheer, E. A. Sete, P. Sivarajah, R. S. Smith, A. Staley, N. Tezak, W. J. Zeng, A. Hudson, B. R. Johnson, M. Reagor, M. P. da Silva, and C. Rigetti, Unsupervised machine learning on a hybrid quantum computer, arXiv:1712.05771.

[17] C. Kokail, C. Maier, R. van Bijnen, T. Brydges, M. K. Joshi, P. Jurcevic, C. A. Muschik, P. Silvi, R. Blatt, C. F. Roos, and P. Zoller, Self-verifying variational quantum simulation of lattice models, Nature (London) 569, 355 (2019).

[18] J. Romero, R. Babbush, J. R. McClean, C. Hempel, P. Love, and A. Aspuru-Guzik, Strategies for quantum computing molecular energies using the unitary coupled cluster ansatz, arXiv: 1701.02691 .

[19] N. Killoran, T. R. Bromley, J. M. Arrazola, M. Schuld, N. Quesada, and S. Lloyd, Continuous-variable quantum neural networks, Phys. Rev. Res. 1, 033063 (2019).

[20] Y. Du, M.-H. Hsieh, T. Liu, and D. Tao, The expressive power of parameterized quantum circuits, Phys. Rev. Res. 2, 033125 (2020).

[21] B. Coyle, D. Mills, V. Danos, and E. Kashefi, The Born supremacy: Quantum advantage and training of an Ising Born machine, npj Quantum Inf. 6, 60 (2020).

[22] J. R. McClean, S. Boixo, V. N. Smelyanskiy, R. Babbush, and $\mathrm{H}$. Neven, Barren plateaus in quantum neural network training landscapes, Nat. Commun. 9, 4812 (2018).

[23] M. Cerezo, A. Sone, T. Volkoff, L. Cincio, and P. J. Coles, Costfunction-dependent barren plateaus in shallow quantum neural networks, arXiv:2001.00550.

[24] J. I. Cirac and P. Zoller, Goals and opportunities in quantum simulation, Nat. Phys. 8, 264 (2012).

[25] P. Hauke, F. M. Cucchietti, L. Tagliacozzo, I. Deutsch, and M. Lewenstein, Can one trust quantum simulators, Rep. Prog. Phys. 75, 082401 (2012).

[26] T. H. Johnson, S. R. Clark, and D. Jaksch, What is a quantum simulator, EPJ Quantum Technol. 1, 10 (2014).

[27] J.-Y. Choi, S. Hild, J. Zeiher, P. Schauß, A. Rubio-Abadal, T. Yefsah, V. Khemani, D. A. Huse, I. Bloch, and C. Gross, Exploring the many-body localization transition in two dimensions, Science 352, 1547 (2016).

[28] J. Bermejo-Vega, D. Hangleiter, M. Schwarz, R. Raussendorf, and J. Eisert, Architectures for Quantum Simulation Showing a Quantum Speedup, Phys. Rev. X 8, 021010 (2018).

[29] X. Gao, Sheng-T. Wang, and L.-M. Duan, Quantum Supremacy for Simulating a Translation-Invariant Ising Spin Model, Phys. Rev. Lett. 118, 040502 (2017).
[30] L. Novo, J. Bermejo-Vega, and R. García-Patrón, Quantum advantage from energy measurements of many-body quantum systems, arXiv:1912.06608.

[31] J. Tangpanitanon, S. Thanasilp, M.-A. Lemonde, N. Dangniam, and D. G. Angelakis, Quantum supremacy in driven quantum many-body systems, arXiv:2002.11946.

[32] A. Parra-Rodriguez, P. Lougovski, L. Lamata, E. Solano, and M. Sanz, Digital-analog quantum computation, Phys. Rev. A 101, 022305 (2020).

[33] A. Martin, L. Lamata, E. Solano, and M. Sanz, Digital-analog quantum algorithm for the quantum Fourier transform, Phys. Rev. Res. 2, 013012 (2020).

[34] A. Galicia, B. Ramon, E. Solano, and M. Sanz, Enhanced connectivity of quantum hardware with digital-analog control, Phys. Rev. Res. 2, 033103 (2020).

[35] D. A. Abanin, E. Altman, I. Bloch, and M. Serbyn, Colloquium: Many-body localization, thermalization, and entanglement, Rev. Mod. Phys. 91, 021001 (2019).

[36] R. Nandkishore and D. A. Huse, Many-body localization and thermalization in quantum statistical mechanics, Annu. Rev. Condens. Matter Phys. 6, 15 (2015).

[37] J. Eisert, M. Friesdorf, and C. Gogolin, Quantum many-body systems out of equilibrium, Nat. Phys. 11, 124 (2015).

[38] M. Rigol, V. Dunjko, and M. Olshanii, Thermalization and its mechanism for generic isolated quantum systems, Nature (London) 452, 854 (2008).

[39] L. D’Alessio, Y. Kafri, A. Polkovnikov, and M. Rigol, From quantum chaos and eigenstate thermalization to statistical mechanics and thermodynamics, Adv. Phys. 65, 239 (2016).

[40] M. Schreiber, S. S. Hodgman, P. Bordia, H. P. Lüschen, M. H. Fischer, R. Vosk, E. Altman, U. Schneider, and I. Bloch, Observation of many-body localization of interacting fermions in a quasirandom optical lattice, Science 349, 842 (2015).

[41] P. Bordia, H. P. Lüschen, S. S. Hodgman, M. Schreiber, I. Bloch, and U. Schneider, Coupling Identical One-Dimensional Many-Body Localized Systems, Phys. Rev. Lett. 116, 140401 (2016).

[42] A. Lukin, M. Rispoli, R. Schittko, M. E. Tai, A. M. Kaufman, S. Choi, V. Khemani, J. Léonard, and M. Greiner, Probing entanglement in a many-body-localized system, Science $\mathbf{3 6 4}$ 256 (2019).

[43] P. Bordia, H. Lüschen, U. Schneider, M. Knap, and I. Bloch, Periodically driving a many-body localized quantum system, Nat. Phys. 13, 460 (2017).

[44] P. Roushan, C. Neill, J. Tangpanitanon, V. M. Bastidas, A. Megrant, R. Barends, Y. Chen, Z. Chen, B. Chiaro, A. Dunsworth, A. Fowler, B. Foxen, M. Giustina, E. Jeffrey, J. Kelly, E. Lucero, J. Mutus, M. Neeley, C. Quintana, D. Sank, A. Vainsencher, J. Wenner, T. White, H. Neven, D. G. Angelakis, and J. Martinis, Spectroscopic signatures of localization with interacting photons in superconducting qubits, Science 358, 1175 (2017).

[45] K. Xu, J.-J. Chen, Y. Zeng, Y.-R. Zhang, C. Song, W. Liu, Q. Guo, P. Zhang, D. Xu, H. Deng, K. Huang, H. Wang, X. Zhu, D. Zheng, and H. Fan, Emulating Many-Body Localization with a Superconducting Quantum Processor, Phys. Rev. Lett. 120, 050507 (2018).

[46] J. Smith, A. Lee, P. Richerme, B. Neyenhuis, P. W. Hess, P. Hauke, M. Heyl, D. A. Huse, and C. Monroe, Many-body 
localization in a quantum simulator with programmable random disorder, Nat. Phys. 12, 907 (2016).

[47] L. D'Alessio and M. Rigol, Long-Time Behavior of Isolated Periodically Driven Interacting Lattice Systems, Phys. Rev. X 4, 041048 (2014).

[48] P. Ponte, Z. Papić, F. Huveneers, and D. A. Abanin, Many-Body Localization in Periodically Driven Systems, Phys. Rev. Lett. 114, 140401 (2015).

[49] D. A. Abanin, W. D. Roeck, and F. Huveneers, Theory of manybody localization in periodically driven systems, Ann. Phys. 372, 1 (2016).

[50] H. Bernien, S. Schwartz, A. Keesling, H. Levine, A. Omran, H. Pichler, S. Choi, A. S. Zibrov, M. Endres, M. Greiner, V. Vuletić, and M. D. Lukin, Probing many-body dynamics on a 51-atom quantum simulator, Nature (London) 551, 579 (2017).

[51] J. Zhang, G. Pagano, P. W. Hess, A. Kyprianidis, P. Becker, H. Kaplan, A. V. Gorshkov, Z. X. Gong, and C. Monroe, Observation of a many-body dynamical phase transition with a 53-qubit quantum simulator, Nature (London) 551, 601 (2017).

[52] M. Raghu, B. Poole, J. Kleinberg, S. Ganguli, and J. SohlDickstein, Proc. Machine Learn. Res. 70, 2847 (2017).

[53] S. Sim, P. D. Johnson, and A. Aspuru-Guzik, Expressibility and entangling capability of parameterized quantum circuits for hybrid quantum-classical algorithms, Adv. Quantum Technol. 2, 1900070 (2019).

[54] I. Glasser, R. Sweke, N. Pancotti, J. Eisert, and J. I. Cirac, Expressive power of tensor-network factorizations for probabilistic modeling, with applications from hidden Markov models to quantum machine learning, Advances in Neural Information Processing Systems 32 Proceedings of the NeurIPS 2019 Conference, https://openreview.net/pdf/ 9ac375d31af73b3f712fee8f782297748f7897f6.pdf.

[55] D. A. Roberts and B. Yoshida, Chaos and complexity by design, J. High Energy Phys. 04 (2017) 121.

[56] A. W. Harrow and A. Montanaro, Quantum computational supremacy, Nature (London) 549, 203 (2017).
[57] L. Stockmeyer, On approximation algorithms for \# P, SIAM J. Comput. 14, 849 (1985).

[58] D. Hangleiter, J. Bermejo-Vega, M. Schwarz, and J. Eisert, Anticoncentration theorems for schemes showing a quantum speedup, Quantum 2, 65 (2018).

[59] J. Haferkamp, D. Hangleiter, A. Bouland, B. Fefferman, J. Eisert, and J. Bermejo-Vega, Closing gaps of a quantum advantage with short-time Hamiltonian dynamics, arXiv:1908.08069.

[60] A. Bouland, B. Fefferman, C. Nirkhe, and U. Vazirani, On the complexity and verification of quantum random circuit sampling, Nat. Phys. 15, 159 (2019).

[61] S. Boixo, S. V. Isakov, V. N. Smelyanskiy, R. Babbush, N. Ding, Z. Jiang, M. J. Bremner, J. M. Martinis, and H. Neven, Characterizing quantum supremacy in near-term devices, Nat. Phys. 14, 595 (2018).

[62] F. Haake, Quantum Signatures of Chaos (Springer International, New York, 2010).

[63] B. Vermersch, A. Elben, M. Dalmonte, J. I. Cirac, and P. Zoller, Unitary $n$-designs via random quenches in atomic Hubbard and spin models: Application to the measurement of Rényi entropies, Phys. Rev. A 97, 023604 (2018).

[64] A. W. Harrow and R. A. Low, Random quantum circuits are approximate 2-designs, Commun. Math. Phys. 291, 257 (2009).

[65] E. Alpaydin, Introduction to Machine Learning (Massachusetts Institute of Technology, Cambridge, 2020).

[66] D. H. Ackley, G. E. Hinton, and T. J. Sejnowski, A learning algorithm for Boltzmann machines, in Readings in Computer Vision, edited by M. A. Fischler and O. Firschein (Morgan Kaufmann, San Francisco, CA, 1987), pp. 522-533.

[67] M. H. Amin, E. Andriyash, J. Rolfe, B. Kulchytskyy, and R. Melko, Quantum Boltzmann Machine, Phys. Rev. X 8, 021050 (2018).

[68] E. R. Anschuetz and Y. Cao, Realizing quantum Boltzmann machines through eigenstate thermalization, arXiv:1903.01359.

[69] For $M=10^{4}$, it takes approximately $1200 \mathrm{CPU}$ hours to generate Fig. 4(b) using Intel Xeon E5-2690 v3 processors. 